\title{
Non-local rheology of dense granular flows
}

\author{
Mehdi Bouzid ${ }^{1,2}$, Martin Trulsson ${ }^{1,3}$, Adrien Izzet ${ }^{1}$, Adeline Favier de Coulomb ${ }^{1}$, Philippe Claudin ${ }^{2, \star}$, Eric Clément $^{1}$, \\ and Bruno Andreotti ${ }^{1}$ \\ ${ }^{1}$ Physique et Mécanique des Milieux Hétérogènes, PMMH UMR 7636 ESPCI - CNRS - Univ. Paris-Diderot - Univ. P.M. Curie, \\ 10 rue Vauquelin, 75005 Paris, France. \\ ${ }^{2}$ Department of Physics, Institute for Soft Matter Synthesis and Metrology, Georgetown University, 37th and O Streets, N.W., \\ Washington, D.C. 20057, USA. \\ ${ }^{3}$ Department of Chemistry, Lund University, PO BOX 124, SE-221 00 Lund, Sweden.
}

\begin{abstract}
The rheology of dense granular flows is studied numerically in a shear cell controlled at constant pressure and shear stress, confined between two granular shear flows. We show that a liquid state can be achieved even far below the yield stress, whose flow can be described with the same rheology as above the yield stress. A non-local constitutive relation is derived from dimensional analysis through a gradient expansion and calibrated using the spatial relaxation of velocity profiles observed under homogeneous stresses. Both for frictional and frictionless grains, the relaxation length is found to diverge as the inverse square root of the distance to the yield point, on both sides of that point. We also make use of a micro-rheometer to determine the influence of a distant shear band on the local rheological behaviour. Finally, we compare various approaches based on different non-local constitutive relations and choices for the fluidity parameter. We emphasise that, to discriminate between the different approaches proposed in the literature, one has to go beyond the predictions derived from linearisation around a uniform stress profile, such as that obtained in a simple shear cell. We argue that future tests can be based on the nature of the chosen fluidity parameter, and the related boundary conditions, as well as the hypothesis made to derive the models and the dynamical mechanisms underlying their dynamics.
\end{abstract}

\section{Introduction}

The physics of granular material has reached today a good level of general understanding [1]. In particular, it is now well established that the rheology of dense granular flows takes a simple form when working at controlled pressure $P$ [2]. It this case, the constitutive laws have been formalised in terms of the inertial number $I=|\dot{\gamma}| d / \sqrt{P / \rho}$, where $\dot{\gamma}$ is the shear rate, $d$ and $\rho$ are the grain size and density [36]. This dimensionless quantity compares the macroscopic shearing time scale $1 / \dot{\gamma}$ to the microscopic time scale of local plastic rearrangements $d / \sqrt{P / \rho}$. In the limit of rigid grains, the effective friction coefficient $\mu$, which compares the shear stress $\tau$ to the pressure $P$, as well as the volume fraction $\phi$ of the system, must be functions of $I$. The shape of the functions $\mu(I)$ and $\phi(I)$ have been determined by means of numerical simulations [4-6, 9], as well as by experimental measurements $[7,8]$. This approach has been successfully applied in different geometries [2, 3], as well as to describe dynamic compressibility effects associated with spontaneous oscillatory motion [11, 12], and this constitutive law is now used as an input in hydrodynamics equations $[10,13]$.

Several experiments have however provided evidence for deviations from the $\mu(I)$-rheology, in the case of heterogeneous systems or when investigating the existence

\footnotetext{
^e-mail: philippe.claudin@espci.fr
}
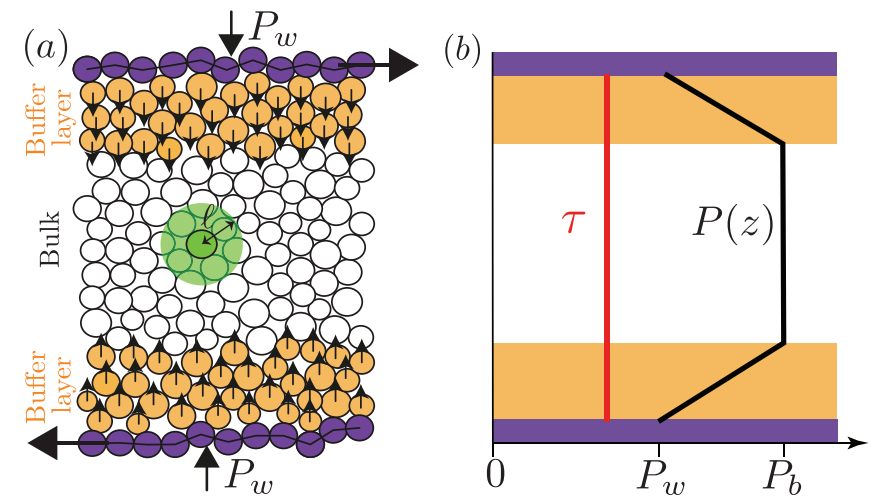

Figure 1. (a) Set-up of the numerical rheometer to probe nonlocal effects. $x$ is the shear direction. The height of the bulk of the cell is $H \simeq 40 d$. The buffer layers are $\Delta \simeq 5 d$ thick. Green zoom: schematic of the influence of grain motion in a neighborhood of size $\ell$ on the local shear rate. (b) Schematic profiles of the pressure $P$ (black line) and of the shear stress $\tau$ (red line) across the cell. In the bulk of the shear cell (white), the pressure is homogeneous: $P=P_{b}$.

of a 'solid-liquid' interface [14-17]. These effects have been qualified as 'non-local', in the sense that flowing at a given location may be enhanced by grain motion in the neighbourhood of that location, so that the $\mu(I)$-rheology 


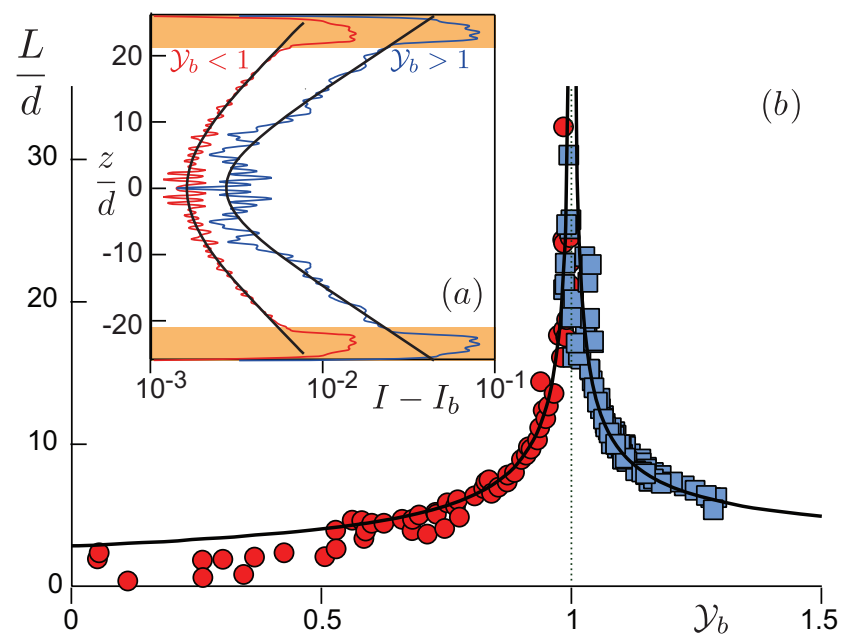

Figure 2. (a) $I$ profiles above (blue line) and below (red line) yield conditions $\mathcal{Y}_{b}=1$. The local rheology predics $I=I_{b}$. Solid black lines: fit of the function $I(z)-I_{b}=\frac{I(H / 2)-I_{b}}{\cosh (H /(2 L))} \cosh (z / L)$, where the relaxation length $L$ is adjusted. (b) $L$ as a function of $\mathcal{y}_{b}$. These data are for frictional grains. A similar behavior is obtained with frictionless grains [22].

with the local values of the parameters is not valid. Nonlocal effects have also been reported in soft glass flows [18-20]. In this case, non-locality has been associated with long range interactions between Eschelby-like elastoplastic events. These events are well defined in the overdamped regime. For inertial flows of rigid grains, however, the picture is different, and the identification of the mechanisms responsible for non-locality is an interesting yet open issue. In this paper, we summarise our recent results on the subject (see also [21]).

\section{A rheometer to probe non-local effects}

We have investigated non-local effects in granular flows by means of DEM numerical simulations [22]. The system is two-dimensional and constituted of $N \simeq 2000$ circular particles of mean diameter $d$, with a $\pm 20 \%$ polydispersity. The shear cell is composed of two rough walls moving along the $x$-direction with opposite velocities (Fig. 1a). These walls are made of the same grains, but glued together. Their position is controlled to ensure a constant normal stress $P_{w}$. Periodic boundary conditions are applied along the $x$-direction. The particle and wall dynamics are integrated using the Verlet algorithm. Contact forces between particles are modeled as linear viscoelastic forces, with a normal contact stiffness large enough $\left(k_{n} / P_{w}>10^{3}\right)$ so that the rigid limit is reached. The tangential spring constant is $k_{t}=0.5 k_{n}$. We have considered both frictionless and frictional grains, with a contact friction coefficient $\mu_{p}=0.4$. The pressure and shear stress in the system are noted $P$ and $\tau$. We define the dimensionless Coulomb yield parameter $\mathcal{Y}=\tau /\left(P \mu_{c}\right)$, where $\mu_{c}$ is the friction coefficient in the zero shear-rate limit, at the jamming volume fraction $\phi_{c}$.
What makes the set-up original is the possibility to impose the profile of the $\mathcal{Y}(z)$ by means of gravity-like forces applied to the grains located in two buffer zones located close to the walls. These forces are oriented downward at the top of the cell, and upward at the bottom. In the bulk of the cell, the pressure is constant $P=P_{b}$. The local rheology predicts in the bulk a linear velocity profile $u_{x}=\dot{\gamma} z$ when $\mathcal{Y}_{b}>1$ corresponding to a finite and constant $I_{b}$, and static grains $\left(u_{x}=0\right.$, corresponding to $\left.I_{b}=0\right)$ when $\boldsymbol{y}_{b}<1$. What we find is that all grains of the system actually move, whatever the value of $\mathcal{Y}_{b}$. The stress ratio $\tau / P$ therefore does not control the liquid-solid transition. Rather than a constant value of the inertial number, we measure $I$ profiles that exponentially relax towards $I_{b}$ over a length $L$ (Fig. 2a). Corresponding velocity profiles follow $u_{x}(z)=\dot{\gamma} z+\frac{u_{x}(H / 2)-\dot{\gamma} H / 2}{\sinh (H /(2 L))} \sinh (z / L)$. The relaxation length $L$ extracted from these profiles is displayed Fig. $2 b$ as a function of $\mathcal{Y}$, showing a divergence on both sides of the local rheology yield point $\mathcal{Y}_{b}=1$ with an exponent $1 / 2$.

\section{A non-local model}

In the usual accessible range of $I$ (between $10^{-4}$ and $10^{-1}$ ), the constitutive relations of the local rheology typically follow the empirical laws

$$
\begin{aligned}
& \mu(I)=\mu_{c}+a I^{\alpha}, \\
& \phi(I)=\phi_{c}-b I^{\alpha},
\end{aligned}
$$

where the exponent $\alpha$ is 0.5 in the frictionless case and 1 in the frictional case. In order to account for non-local effects, we perform a gradient expansion of the functional $\mathcal{Y}[I]$. Assuming that non-locality results from a statistically isotropic short-range interaction between shear zones (Fig. 1b), the lowest order operator is the Laplacian $\nabla^{2} I$. As a direct consequence, $I$ and its gradient must be continuous. Furthermore, we assume that the non-local correction remains finite as $I \rightarrow 0$, so that the expansion must be expressed in terms of $\kappa \equiv \ell^{2}\left(\nabla^{2} I\right) / I$, where $\ell$ is a length on the order of few grain diameters (Fig. 1a). At the linear order in $\kappa$, the constitutive relation is then :

$$
\mathcal{y}=\frac{\mu(I)}{\mu_{c}}(1-\kappa) .
$$

$\kappa$ is positive when the point considered is surrounded by a more liquid region (higher $I$ ). This region flows more easily than expected from the local value of $I$, so that the corresponding shear stress is lower.

Linearising the rheology around $I_{b}$ above yielding conditions, we find a second order differential equation, whose solutions are exponentials (hence sinh shapes for the velocity profiles), with a relaxation length $L_{>}=$ $\ell\left[\mathcal{Y}_{b} / \alpha\left(\mathcal{Y}_{b}-1\right)\right]^{1 / 2}$. Below yielding conditions, the nonlocal correction is of already of zeroth order, similarly leading to a relaxation length $L_{<}=\ell\left[1 /\left(\boldsymbol{Y}_{b}-1\right)\right]^{1 / 2}$. These expressions quantitatively fit the data shown in Fig. $2 \mathrm{~b}$, with $\ell \simeq 3 d$. Further evidence for the squareroot divergence, with log-log plots for both frictional and frictionless cases, can be found in [22]. Importantly, the 


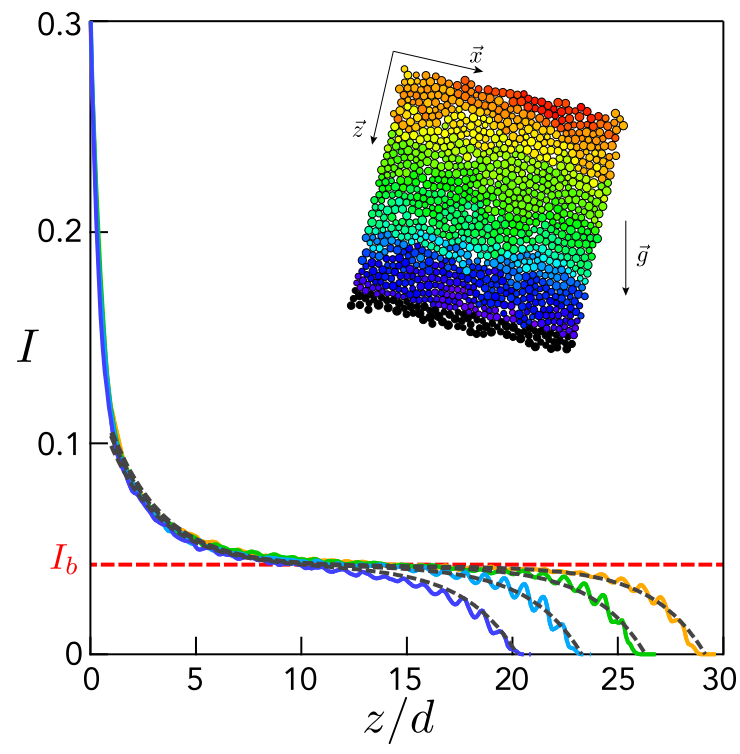

Figure 3. Profiles of the inertial number $I(z)$ for gravity flows of different thickness (dark blue: $H \simeq 20 d$, light blue: $H \simeq 23 d$, green: $H \simeq 26 d$, yellow: $H \simeq 28 d$, ) at a given inclination $\theta \simeq 18^{\circ}$. The free surface is at $z=0$. Red dashed line: prediction from the local rheology. Black dashed lines: fits of the non-local model with the function $I=I_{b}+C_{1} \exp (z / L)+C_{2} \exp (-z / L)$. These data are for frictional grains $\left(\mu_{p}=0.5\right)$. A similar behavior is obtained with frictionless grains. Inset: schematic of the incline plane. Color code for the velocity of the grains along the $\vec{x}$ axis.

divergence of $L$ is not the signature of a dynamic phase transition controlled by $\mathcal{Y}$. Both above and below yielding conditions, the system is constituted of a single liquid phase, with permanent fluctuations, in contrast to a solid that would flow by means of elasto-plastic events. The situation would of course be different looking at the initial shearing of a granular packing prepared in a noncritical solid state, where 'hot spots' corresponding to localised plastic events are visualised [23]. Here the stationary shearing maintains the liquid state in the whole system.

Importantly, one should realise that the validity of nonlocal constitutive relation is not restricted to the description of a creeping flow dynamics, and suited to go beyond the flow blockade as predicted by a local $\mu(I)$ rheology. But also, it is able to reproduce quantitatively flow profiles in inhomogeneous situations, which is actually of a much more general interest. This is illustrated in Fig. 3, in a gravity flows situation where the local rheology would predicts a constant inertia number $I_{b}=0.4$. The actual result of the numerical simulation shows that the $I(z)$ profile is indeed reproduced quantitatively by the non-local rheology.

\section{Discussion}

Other approaches have been proposed to model nonlocality in granular flows (see [21] and references therein). For example, Pouliquen and Forterre have proposed a formulation based on an analogy with Eyring's transition state theory for the viscosity of liquids, where mechanical fluctuations would play the role of temperature in thermal systems [24]. Another example is that of Rognon et al.[25]. Closer to our framework, Kamrin et al. have proposed a model [26-28] directly adapted from the Kinetic Elasto-Plastic theory introduced by Bocquet et al. [29] for soft matter.

A crucial issue is the choice of the quantity, called the fluidity $f$, for which the non-local constitutive relation is written. The fluidity must vanish in the solid phase and increase with the ability to flow. Here we have made the simplest choice in the context of the $\mu(I)$-rheology and take it as the inertial number itself $f=I$. As for comparison, Kamrin et al. rather took $f=\dot{\gamma} / \tau$ for the fluidity parameter. The choice for $f$ is a central issue, which has strong physical consequences: boundary and continuity conditions will apply on $f$ and its gradient.

Interestingly, Kamrin et al.'s approach and our model lead to the same predictions for the velocity profile in a situation where the stress is homogeneous, such that the shear cell described above. The differences between the approaches must be tested in situations that are strongly heterogeneous in space. In this spirit, we have performed numerical simulations where a secondary microrheometer is placed in the bulk of a shear cell as presented above [30]. Shearing within the micro-rheometer is obtained by means of localised bulk forces along two lines which induce a discontinuity of the shear stress. The pressure remains constant and the direction of shearing is reversed ( $\dot{\gamma}$ changes sign at the discontinuity). We find that the local shear rate is proportional to that in the bulk and decreases roughly exponentially with the distance to the yield conditions. We finally measured numerically the ratio of the absolute value of the shear rate on one side and on the other side of the stress discontinuity, and shown that $|\dot{\gamma}|$ is indeed continuous, unlike $\dot{\gamma} / \tau$.

In conclusion, in spite of some success in the framework of simple rheological situations, a better clarification of the microscopic mechanisms responsible for the nonlocality is needed to move forwards and provide a fully consistent picture of the constitutive relation hence proposed. We have for example explored the role of particle softness in the strain mode [21,31]. Furthermore, to make such a theory fully practical, one should provide a meaningful determination of the boundary conditions, for example with rough-walls, using parameters derived on physical grounds and not from an a-posteriori fitting of the flow curves as it is currently done. This question is under current investigation.

\section{Acknowledgements}

BA is supported by Institut Universitaire de France. This work was funded by the ANR JamVibe and by a CNES grant.

\section{References}

[1] B. Andreotti, Y. Forterre and O. Pouliquen, Granular Media, Between Fluid and Solid, Cambridge University 
Press (2013).

[2] Y. Forterre and O. Pouliquen, Ann. Rev. Fluid Mech. 40, 1-24 (2008).

[3] GDR MiDi, Eur. Phys. J. E 14, 341-365 (2004).

[4] F. da Cruz, S. Emam, M. Prochnow , J.-N. Roux and F. Chevoir, Phys. Rev. E 72, 021309 (2005).

[5] P. Jop, Y. Forterre and 0. Pouliquen, Nature 441, 727730 (2006).

[6] T. Hatano, Phys. Rev. E 75, 060301(R) (2007).

[7] P. Jop, Y. Forterre and O. Pouliquen, J. Fluid Mech. 541, 167-192 (2005).

[8] A. Fall, G. Ovarlez, D. Hautemayou, C. Mézière, J.N. Roux and F. Chevoir, J. Rheology 59, 1065-1080 (2015).

[9] E. Azéma and F. Radjaï, Phys. Rev. Lett. 112, 078001 (2014).

[10] T. Barker, D.G. Schaeffer, P. Bohorquez and J.M.N.T. Gray, J. Fluid Mech. 779, 794-818 (2015).

[11] L. Staron, Phys. Rev. E 86, 041307 (2012).

[12] M. Trulsson, M. Bouzid, P. Claudin and B. Andreotti, Europhys. Lett. 103, 38002 (2013).

[13] P.-Y. Lagrée, L. Staron and S. Popinet, J. Fluid. Mech. 686, 378-408 (2011).

[14] T.S. Komatsu, S. Inagaki, N. Nakagawa and S. Nasuno, Phys. Rev. Lett. 86, 1757 (2001).

[15] I.S. Aranson, L.S. Tsimring, F. Malloggi and E. Clément, Phys.Rev.E 78, 031303 (2008).

[16] K.A. Reddy, Y. Forterre and O. Pouliquen, Phys. Rev. Lett. 106, 108301 (2011).
[17] E. Wandersman, M. Van Hecke, Europhys. Lett. 105, 24002 (2014).

[18] J. Goyon, A. Colin, G. Ovarlez, A. Ajdari and L. Bocquet, Nature. 454, 84 (2008).

[19] J.-L. Barrat and A. Lemaître, In 'Dynamical heterogeneities in glasses, colloids and granular media', 150, 264 (2011).

[20] V. Mansard and A. Colin, Soft Matter 8, 4025 (2012).

[21] M. Bouzid, A. Izzet, M. Trulsson, E. Clément, P. Claudin and B. Andreotti, Eur. Phys. J. E 38, 125 (2015).

[22] M. Bouzid, M. Trulsson, P. Claudin, E. Clément and B. Andreotti, Phys. Rev. Lett. 111, 238301 (2013).

[23] A. Amon, V.B. Nguyen, A. Bruand, J. Crassous, E. Clément, Phys. Rev. Lett. 108, 135502 (2012).

[24] O. Pouliquen and Y. Forterre, Phil. Trans. R. Soc. A. 367, 5091 (2009).

[25] P.G. Rognon, T. Miller, B. Metzger and I. Einav, J. Fluid Mech. 764, 171 (2015).

[26] K. Kamrin and G. Koval, Phys. Rev. Lett. 108, 178301 (2012).

[27] D.L. Henann and K. Kamrin, Proc. Natl. Acad. Sci. USA 110, 6730 (2013).

[28] D.L. Henann and K. Kamrin, Phys. Rev. Lett. 113, 178001 (2014).

[29] L. Bocquet, A. Colin and A. Ajdari, Phys. Rev. Lett. 103, 036001 (2009).

[30] M. Bouzid, M. Trulsson, P. Claudin, E. Clément and B. Andreotti, Europhys. Lett. 109, 24002 (2015).

[31] A. Favier de Coulomb, M. Bouzid, P. Claudin, E. Clément and B. Andreotti, Submitted. 